\title{
Phosphodiesterase 2 Inhibitors Promote Axonal Outgrowth in Organotypic Slice Co-Cultures
}

\author{
C. Heine ${ }^{a, b} \quad$ K. Sygnecka ${ }^{a, b} \quad$ N.Scherfc,d $\quad$ A. Berndt ${ }^{e} \quad$ U. Egerland ${ }^{\text {e }} \quad$ T. Hage ${ }^{e}$ \\ H. Franke ${ }^{b}$ \\ aTranslational Centre for Regenerative Medicine (TRM), ${ }^{b}$ Rudolf Boehm Institute of Pharmacology and Toxicology, \\ and 'Interdisciplinary Centre for Bioinformatics, University of Leipzig, Leipzig, dInstitute for Medical Informatics \\ and Biometry, Dresden University of Technology, Dresden, and ebiocrea, Radebeul, Germany
}

\section{Key Words \\ Axonal outgrowth - Biocytin - Development • \\ Dopaminergic system - Organotypic slice co-culture • \\ Phosphodiesterase $\cdot$ Regeneration $\cdot$ Repair · Tracing • \\ Tyrosine hydroxylase}

\begin{abstract}
The development of appropriate models assessing the potential of substances for regeneration of neuronal circuits is of great importance. Here, we present procedures to analyze effects of substances on fiber outgrowth based on organotypic slice co-cultures of the nigrostriatal dopaminergic system in combination with biocytin tracing and tyrosine hydroxylase labeling and subsequent automated image quantification. Selected phosphodiesterase inhibitors (PDE-Is) were studied to identify their potential growth-promoting capacities. Immunohistochemical methods were used to visualize developing fibers in the border region between ventral tegmental area/substantia nigra co-cultivated with the striatum as well as the cellular expression of PDE2A and PDE10. The quantification shows a significant increase of fiber density in the border region induced by PDE2-Is (BAY607550; ND7001), comparable with the potential of the nerve growth factor and in contrast to PDE10-I (MP-10). Analysis of
\end{abstract}

\begin{tabular}{|c|c|}
\hline KARGER & $\begin{array}{ll}\text { (c) } 2012 \text { S. Karger AG, Basel } & \text { Karger } \\
1424-862 X / 13 / 0214-0197 \$ 38.00 / 0 & \text { Open access }\end{array}$ \\
\hline $\begin{array}{l}\text { E-Mail karger@karger.com } \\
\text { www.karger.com/nsg }\end{array}$ & $\begin{array}{l}\text { This is an Open Access article licensed under the terms of the } \\
\text { Creative Commons Attribution-NonCommercial 3.0 Un- } \\
\text { ported license (CC BY-NC) (www.karger.com/OA-license), } \\
\text { applicable to the online version of the article only. Distribu- } \\
\text { tion permitted for non-commercial purposes only. }\end{array}$ \\
\hline
\end{tabular}

tyrosine hydroxylase-positive fibers indicated a significant increase after treatment with BAY60-7550 and nerve growth factor in relation to dimethyl sulfoxide. Additionally, a dosedependent increase of intracellular cGMP levels in response to the applied PDE2-Is in PDE2-transfected HEK293 cells was found. In summary, our findings show that PDE2-Is are able to significantly promote axonal outgrowth in organotypic slice co-cultures, which are a suitable model to assess growth-related effects in neuro(re)generation.

Copyright $\odot 2012$ S. Karger AG, Base

\section{Introduction}

Axonal projections and the basic organization of midbrain dopaminergic (DAergic) neurons are the subject of interest when studying human neurological disorders, e.g. Parkinson's disease [1], schizophrenia [2], and drug abuse or addiction $[3,4]$. The mesencephalon as a region of complex anatomical organization, and projection patterns of the DAergic neurons are divided into the retrorubral area

C.H. and K.S. contributed equally to the study.
PD Dr. Heike Franke

Rudolf Boehm Institute of Pharmacology and Toxicology

University of Leipzig, Härtelstrasse 16-18

DE-04107 Leipzig (Germany)

Tel. +49 341972 4602, E-Mail Heike.Franke@medizin.uni-leipzig.de 
(A8 cell group), substantia nigra (SN; A9 cell group), and the ventral tegmental area (VTA; A10 cell group) [5-7]. Tract-tracing techniques have revealed three projections to forebrain targets, namely the mesolimbic, the mesocortical, and the nigrostriatal pathways (for references, see [8]). The trajectories from the SN and VTA target all intrinsic nuclei of the basal ganglia while exhibiting preferential concentrations of terminals in the dorsal and ventral striatum (STR) $[1,8]$. The balance between these projections is thought to be regulated by afferent DAergic signals from the VTA and SN pars compacta, acting on differentially distributed D1 and D2 dopamine (DA) receptors, e.g. on striatal medium spiny neurons. The activation of D1 receptors leads to an intracellular accumulation of cyclic adenosine $3^{\prime}, 5^{\prime}$-monophosphate (cAMP) via the adenylyl cyclase and following activation of cAMP-dependent protein kinase. Otherwise, DA inhibits the adenylyl cyclase via D2 receptors (for references, see [9]).

It is well known that disruption of DAergic cell activity and the loss of ascending projections to the basal ganglia results in the emergence of fundamental pathological features. Due to the necessity for therapeutic strategies promoting neuro(re)generation, there is a clear need to develop appropriate test systems to assess the potential of different substances regarding regeneration and repair of neuronal circuits. Over the last years, we established ex vivo models of organotypic slice co-cultures of the DAergic system [10-12]. Parts of this system were reconstructed using tissue slices from the VTA/SN and STR or the prefrontal cortex, respectively, to analyze the cytoarchitectural organization of the VTA/SN and the innervations of the target regions by DAergic fibers. Our results demonstrated that tyrosine hydroxylase (TH)-immunopositive neurons are also able to develop their characteristic innervation patterns in organotypic slice co-cultures [10]. A number of intracellular and extracellular molecules are involved in the regulation of neuronal differentiation. Using our model system, we were able to identify a trophic support in axonal outgrowth after purinergic stimulation $[11,12]$. Moreover, second messengers cAMP and cyclic guanosine $3^{\prime}, 5^{\prime}$-monophosphate (cGMP), formed from the triphosphates ATP and GTP, appear to play prominent roles in regulating neuronal differentiation and neuroplasticity $[13,14]$. Elevated intracellular levels of the cyclic nucleotides are important for axon regeneration, even beyond the required increase in cAMP levels needed to respond to most factors that support cell survival [15]. Cyclic nucleotide phosphodiesterases (PDEs) comprise a superfamily of metallophosphohydrolases that specifically cleave the $3^{\prime}, 5^{\prime}$-cyclic phosphate moiety of cAMP and/or cGMP and terminate the action of cyclic nucleotide signaling [16]. Eleven individual PDE subfamilies (PDE1-PDE11) with varying selectivity for cAMP or cGMP have been identified in mammalian tissues based on sequence similarities, inhibitor sensitivity, and biochemical properties. They are involved in mediating a range of different functions, including cytoskeletal rearrangement, gene transcription, and regulation of ion channel function $[17,18]$. Recent data have shown that PDE2 and PDE10 are localized in the nigrostriatal system and hydrolyze both cAMP and cGMP. The inhibition of PDE2 selectively increases cyclic nucleotide levels, influencing synaptic plasticity and memory formation $[19,20]$. Inhibition of PDE10 modulates neuronal activity within the striatopallidal and nigrostriatal pathway and leads to efficacy in behavioral models predicting an antipsychotic effect [21-24].

The aim of the present study was to characterize possible trophic/regenerative properties of selected PDE2 inhibitors (PDE2-Is; BAY60-7550 and ND7001) and PDE10I (MP-10), as compared to the effect of the neurotrophin nerve growth factor (NGF) by using an organotypic slice co-culture model (VTA/SN+STR). To quantify the potential of stimulating fiber growth, density of neuronal fibers in the border region of slice co-cultures was determined using both biocytin-tracing technique and $\mathrm{TH}$ immunolabeling, and subsequent automated image quantification.

\section{Materials and Methods}

\section{Materials/Substances}

Selected PDE-Is with different subclass specificities were used (details, see table 1; structural formula, see fig. 1): BAY60-7550 (2-(3,4-dimethoxybenzyl)-7-\{(1R)-1-[(1R)-1-hydroxyethyl]-4phenyl-butyl\}-5-methyl imidazo[5,1-f][1,2,4]triazin-4(3H)-one; Alexis Biochemicals, San Diego, Calif., USA), ND7001 (3-(8-methoxy-1-methyl-2-oxo-7-phenyl-2,3-dihydro-1H-benzo[e][1,4]diazepin-5-yl)-benzamide), and MP-10 (2-[4-(1-methyl-4-pyridin4-yl-1H-pyrazol-3-yl)-phenoxymethyl]-quinoline 3) were synthesized at biocrea (Radebeul, Germany).

Furthermore, the following substances/factors were applied: NGF (Sigma-Aldrich, Taufkirchen, Germany), dimethyl sulfoxide (DMSO; AppliChem GmbH, Darmstadt, Germany), artificial cerebrospinal fluid (ACSF) of the composition (mM): $126 \mathrm{NaCl}$; $2.5 \mathrm{KCl} ; 1.2 \mathrm{NaH}_{2} \mathrm{PO}_{4} ; 1.3 \mathrm{MgCl}_{2}$, and $2.4 \mathrm{CaCl}_{2}$ (pH 7.4; Hospital Pharmacy, University of Leipzig, Germany).

\section{In vitro PDE Assay}

$\mathrm{PDE}$ activity was measured by the conversion of $\left[{ }^{3} \mathrm{H}\right]$-cAMP and $\left[{ }^{3} \mathrm{H}\right]-c G M P$ into $\left[{ }^{3} \mathrm{H}\right]-A M P$ and $\left[{ }^{3} \mathrm{H}\right]-\mathrm{GMP}$, respectively, as described previously [25]. PDEs 1B, 2A, 3A, 4A, 5A, 7B, 8A, 9A, $10 \mathrm{~A}$, and $11 \mathrm{~A}$ were generated from full-length human recombi- 
Fig. 1. Chemical structures of the PDE substrates cGMP and cAMP and of the PDE-Is BAY60-7550, ND7001, and MP-10.

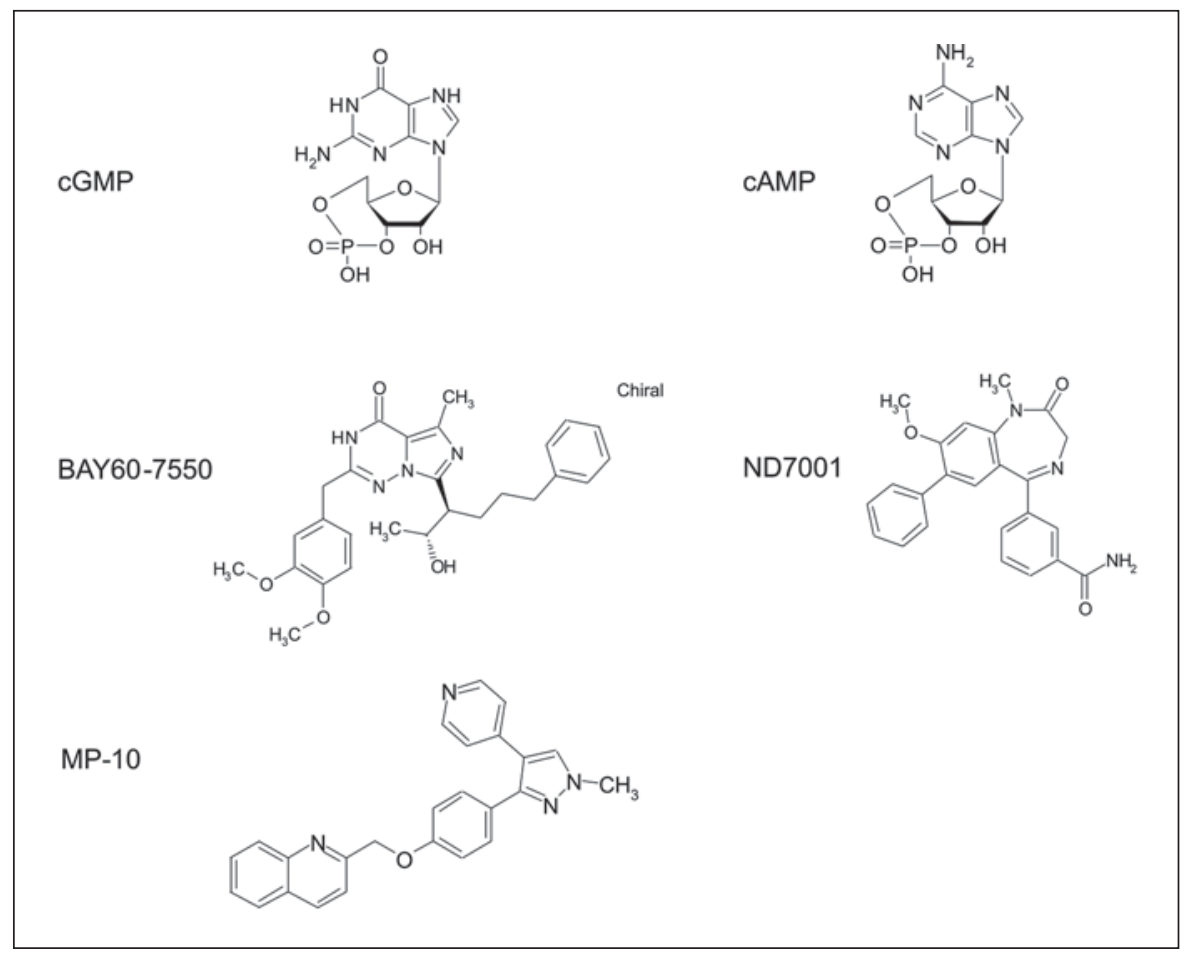

nant clones. PDE6 was isolated from bovine retina as described previously [26]. PDE activity was measured with the preferred substrates, in detail, for PDE1B, 2A, 3A, 4A, 7B, 8A, 10A, and 11A cAMP was used and for PDE5A, 6, and 9A cGMP, at or below the $\mathrm{K}_{\mathrm{m}}$ value (Michaelis-Menten constant). PDE1B and PDE2A were activated by $\mathrm{Ca}^{2+} /$ calmodulin and by cGMP, respectively. The other PDEs did not need any additional activation procedure. Inhibition of PDE activity was measured using a scintillation proximity assay at varied compound concentrations and optimized fixed enzyme concentrations for each enzymatic assay. $\mathrm{IC}_{50}$ (half maximal inhibitory concentration) values were calculated with the Hill2 parameter model from at least four independent experiments, done in duplicates.

\section{Intracellular cGMP Assay}

The selected PDE-Is BAY60-7550 and ND7001 were tested in a cellular test system to analyze the effects on intracellular cGMP levels. Human embryonic kidney (HEK) 293 cells, stably transfected with the full-length sequence of human PDE2A (NM002599), were cultured in collagen I-coated 96-well plates (70,000 cells/well) over night. On the next day, the test compounds were administered at a final DMSO concentration of $0.5 \%$ and incubated for $30 \mathrm{~min}$ at $37^{\circ} \mathrm{C}$ and $5 \% \mathrm{CO}_{2}$. This was followed by incubation with the guanylyl cyclase (GC)-activating agent sodium nitroferricyanide(III) dihydrate (sodium nitroprusside, SNP, $100 \mu \mathrm{M}$; Sigma-Aldrich) for $10 \mathrm{~min}$. Wells incubated without test compound in the presence and absence of SNP were used as controls for statistical analysis. The level of intracellular cGMP was determined with the enzyme-linked immunosorbent assay system (cGMP EIA System; GE Healthcare UK Ltd., Little Chalfont, England).

Phosphodiesterase 2 Inhibitors Promote Axonal Outgrowth
Table 1. IC50 values (nM) of the PDE2-Is BAY60-7550 and ND7001, and the PDE10-I MP-10 against individual phosphodiesterases

\begin{tabular}{lccc}
\hline PDE & BAY60-7550 & ND7001 & MP-10 \\
\hline hPDE2A & 0.18 & 57 & 2,630 \\
hPDE1B & 426 & $>5,000$ & $>5,000$ \\
hPDE3A & $>5,000$ & $>10,000$ & $>5,000$ \\
hPDE4A & 2,130 & $>10,000$ & 2,230 \\
hPDE5A & 516 & $>10,000$ & $>5,000$ \\
bPDE6 & 2,200 & $>10,000$ & $>5,000$ \\
hPDE7B & 1,740 & $>10,000$ & $>5,000$ \\
hPDE8A & $>5,000$ & $>10,000$ & $>5,000$ \\
hPDE9A & $>5,000$ & $>10,000$ & $>5,000$ \\
hPDE10A & 1,640 & 1,460 & 1.14 \\
hPDE11A & $>5,000$ & $>10,000$ & $>5,000$ \\
\hline
\end{tabular}

Overview of the $\mathrm{IC}_{50}$ values (nM) of the PDE2-Is BAY60-7550 and ND7001, and the PDE10-I MP-10 against individual human (h) PDEs as well as the bovine (b) PDE6.

\section{Animals}

Neonatal rat pups (WISTAR RjHan, own breed; animal house of the Rudolf Boehm Institute of Pharmacology and Toxicology, University of Leipzig) of postnatal day 1-5 (P1-5) were used for preparation of the organotypic slice co-cultures. The animals were housed under standard laboratory conditions, under a 12- 
hour light/12-hour dark cycle and allowed access to lab food and water ad libitum.

All of the animal use procedures were approved by the Committee of Animal Care and Use of the relevant local governmental body in accordance with the law of experimental animal protection.

\section{Preparation of Slice Cultures}

Slice co-cultures were prepared from P1-5 old rats and cultivated according to the 'static' culture protocol described by Franke et al. [10] (for details, see also fig. 2). Briefly, rat pups were decapitated and the brains were removed from the skull under sterile conditions. The brains were apposed to an agar block and fixed onto the specimen stage of a slicer (Leica VT 1000S; Nussloch, Germany) with cyanoacrylate glue (Histoacryl ${ }^{\circledR}$; B. Braun, Tuttlingen, Germany). Coronal sections (thickness $300 \mu \mathrm{m}$ ) were cut at mesencephalic (fig. 2: A) and forebrain levels (fig. 2: B). During the preparation of organotypic slice cultures of the ventral mesencephalon we did not attempt to separate the VTA and the SN. For further discussion, this area will be named VTA/SN. After preparation of the VTA/SN (fig. 2: A', asterisk) and the STR (fig. 2: $\mathrm{B}$, asterisk), respectively, the slices were transferred into petri dishes filled with cold $\left(4^{\circ} \mathrm{C}\right)$ preparation solution (MEM, Minimum Essential Medium, $2 \mathrm{~mm}$ glutamine; Invitrogen $\mathrm{GmbH}$, Darmstadt, Germany) supplemented with the antibiotic gentamicin $(50 \mu \mathrm{g} / \mathrm{ml}$; Invitrogen $\mathrm{GmbH})$. Selected sections were placed next to each other on moistened translucent membrane inserts (0.4 $\mu \mathrm{m}$; Millicell-CM, Millipore, Bedford, Mass., USA) as cocultures (VTA/SN+STR) and were put in 6-well plates each filled with $1 \mathrm{ml}$ incubation medium (50\% MEM, 25\% Hank's Balanced Salt Solution, $25 \%$ heat inactivated horse serum; glutamine to a final concentration of $2 \mathrm{mM}$; all from Invitrogen $\mathrm{GmbH}$ and with $0.044 \%$ sodium hydrogen carbonate, $\mathrm{NaHCO}_{3}$; Sigma-Aldrich). The $\mathrm{pH}$ was adjusted to 7.2 . The cultures were stored at $37^{\circ} \mathrm{C}$ in $5 \% \mathrm{CO}_{2}$ and the medium was changed 3 times a week.

\section{Fixation of the Tissues}

After 10 days in vitro (DIV), the cultures were fixed in a solution consisting of $4 \%$ paraformaldehyde, $0.1 \%$ glutaraldehyde, $15 \%$ picric acid in phosphate buffer ( $\mathrm{PB}, 0.1 \mathrm{M}$; $\mathrm{pH} 7.4)$ for $2 \mathrm{~h}$ and subsequently washed with $\mathrm{PB}$ intensively. Following fixation, the co-cultures were cut into 50 - $\mu \mathrm{m}$ thick slices by means of the vibratome.

\section{Immunohistochemistry}

(a) Qualitative Characterization of PDEs. Following pre-incubation in $1 \% \mathrm{H}_{2} \mathrm{O}_{2}$ solution for $25 \mathrm{~min}$ as well as in blocking solution containing $10 \%$ normal horse serum in $0.1 \mathrm{M}$ PB for $1 \mathrm{~h}$, respectively, the slices were incubated with goat anti-PDE2A (1:100; Santa Cruz Biotechnology, Inc., Santa Cruz, Calif., USA) or rabbit anti-PDE10 (1:500; FabGennix Inc., Frisco, Tex., USA) for $48 \mathrm{~h}$ at $4^{\circ} \mathrm{C}$.

(b) Quantitative Characterization of TH-Positive Fibers. After pre-treatment with $\mathrm{H}_{2} \mathrm{O}_{2}$ and incubation with blocking solution, the slices were incubated with mouse anti-TH $(1: 1,000$; Chemicon, Temecula, Calif., USA) for $48 \mathrm{~h}$ at $4^{\circ} \mathrm{C}$.

$(a, b)$ After washing with $\mathrm{PB}$, the slices were incubated with biotinylated anti-goat, biotinylated anti-rabbit or biotinylated anti-mouse immunoglobulin IgG $(\mathrm{H}+\mathrm{L})(1: 65$; Vector Laboratories, Inc., Burlingame, Calif., USA), respectively, for $2 \mathrm{~h}$ at room tem-

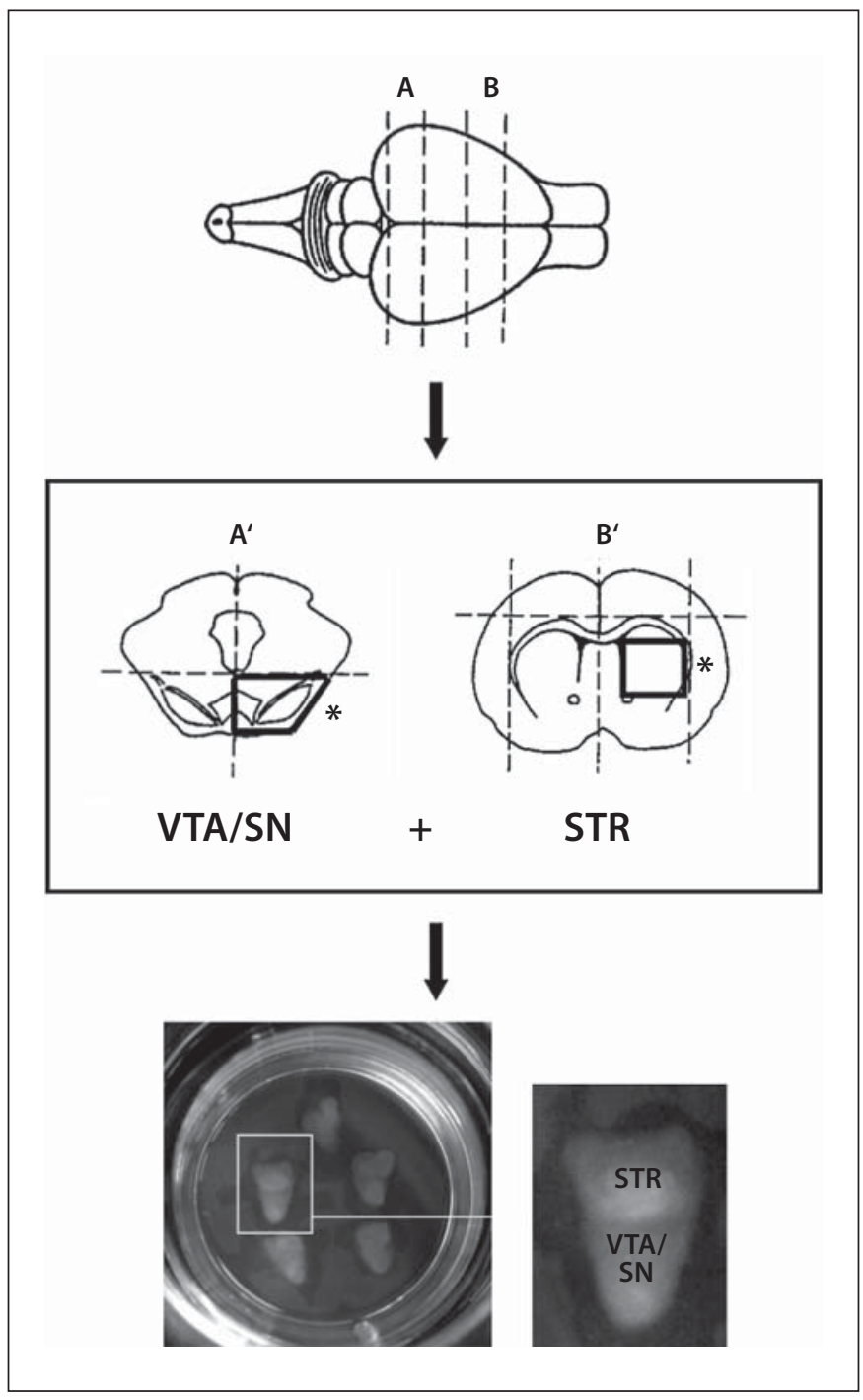

Fig. 2. Schematic illustration of the preparation procedure to obtain organotypic slice co-cultures of the DAergic system as described previously by Franke et al. [10]. Intact brains were removed from 1- to 5-day-old rats. Afterwards, transverse cuts were made at the level of A to isolate the mesencephalon and the striatal region (B). Additional horizontal cuts were made indicated by the dashed lines to separate the VTA/SN $\left(A^{\prime} *\right)$ and the STR $\left(B^{\prime}{ }^{*}\right)$. In the last step, selected coronal sections (thickness $300 \mu \mathrm{m}$ ) were placed next to each other on moistened membranes as co-cultures as shown in the pictures.

perature. Afterwards, the avidin-biotin-horseradish peroxidase complex (1:50; ABC-Elite Kit, Vector Laboratories, Inc.) was applied and visualized by 3,3'-diaminobenzidine hydrochloride (DAB; Sigma-Aldrich) as the chromogen. For the TH immunolabeling, nickel/cobalt-intensified DAB was used. After mounting on glass slides, the stained slices were dehydrated in a series of graded ethanol, processed through n-butylacetate and coverslipped with Entellan (Merck, Darmstadt, Germany). 


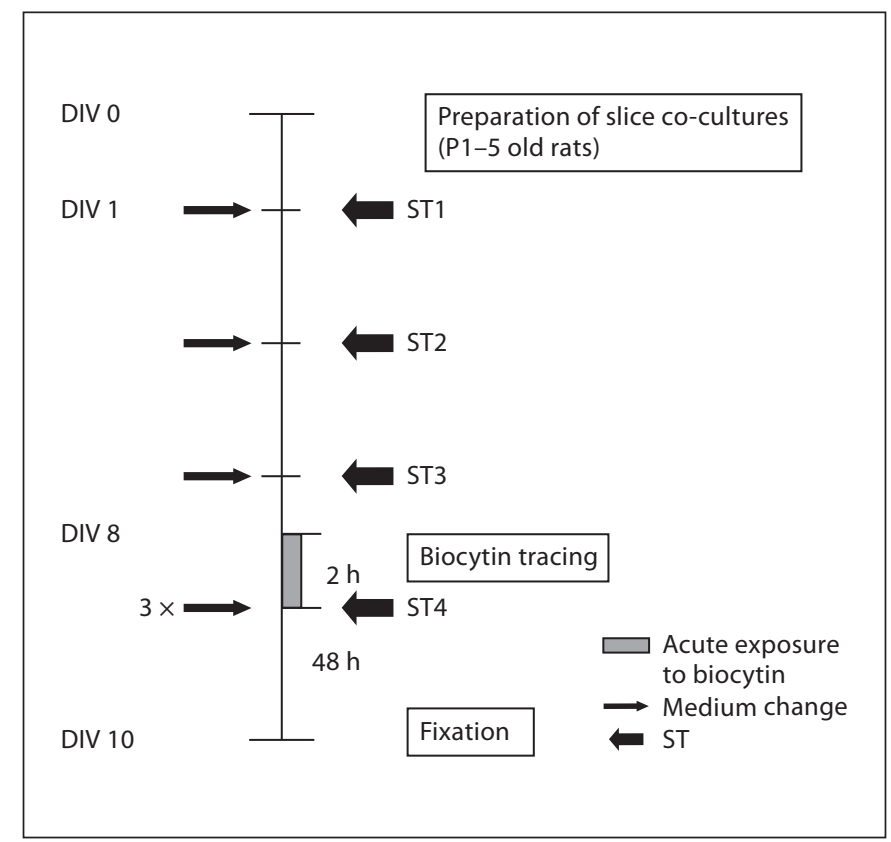

Fig. 3. A detailed chronological scheme of the experimental procedure is illustrated. During the incubation period of the cultures, the treatment was executed 4 times (ST), starting on DIV 1 using the respective substances. At DIV 8, the biocytin tracing was performed as described and, finally, at DIV 10, the co-cultures were fixed and analyzed. ST = Substance treatment.

\section{Immunofluorescence}

Single Labeling. After washing with Tris-buffered saline (TBS, $0.05 \mathrm{M} ; \mathrm{pH}$ 7.6) and blocking with TBS containing fetal calf serum (FCS, 5\%) and Triton X-100 (0.3\%), the slices were incubated with mouse anti-TH (1:1,000; Chemicon); mouse anti- $\beta$ III-tubulin (1:400; Promega GmbH, Mannheim, Germany) or mouse antiglial fibrillary acidic protein (GFAP, 1:1,000; Sigma-Aldrich), respectively, for $48 \mathrm{~h}$ at $4{ }^{\circ} \mathrm{C}$. The visualization of the respective primary antibodies was performed with carbocyanine (Cy)2-conjugated goat anti-mouse IgG (1:400; Jackson ImmunoResearch, West Grove, Pa., USA). In addition, slices were stained with the nucleic acid probe Hoechst 33342 (Hoe, final concentration $40 \mu \mathrm{g} /$ ml; Molecular Probes, Leiden, The Netherlands) to identify the cell nuclei.

Multiple Fluorescence Labeling. After the blocking step, performed as described above, the slices were incubated with an antibody mixture of rabbit anti-microtubule-associated protein-2 (MAP2, 1:500; Millipore, Temecula, Calif., USA), mouse antiGFAP (1:1,000; Sigma-Aldrich) and goat anti-PDE2A (1:100; Santa Cruz Biotechnology, Inc.) or mouse anti-MAP2 (1:1,000; Millipore), goat anti-GFAP (1:300; Santa Cruz Biotechnology, Inc.) and rabbit anti-PDE10 (1:500; FabGennix Inc.), respectively, in TBS-containing 5\% FCS and $0.3 \%$ Triton X-100 for $48 \mathrm{~h}$ at $4{ }^{\circ} \mathrm{C}$. The simultaneous visualization of the different primary antisera was performed with a mixture of secondary antibodies specific for the appropriate species IgG (rabbit, mouse, goat; all from Jackson ImmunoResearch). In detail, Cy2- (1:400), Cy3- (1:1000), and
Cy5- (1:100) conjugated IgGs were diluted in TBS supplemented with 5\% FCS and $0.3 \%$ Triton X-100 and the mixture was applied for $2 \mathrm{~h}$ at room temperature.

$\mathrm{TH}$-double immunofluorescence was performed using antibodies against mouse anti-TH (1:1,000; Chemicon) and goat antiPDE2A (1:100; Santa Cruz Biotechnology, Inc.) or rabbit antiPDE10 (1:500; FabGennix Inc.), respectively, in TBS-containing $5 \%$ FCS and $0.3 \%$ Triton X-100 for $48 \mathrm{~h}$ at $4^{\circ} \mathrm{C}$. The visualization was performed with a mixture of DyLight ${ }^{\mathrm{TM}} 649$-conjugated donkey anti-mouse IgG (1:100, color coded in green) and Cy3-conjugated donkey anti-rabbit or anti-goat (1:1,000; Jackson ImmunoResearch, each). Subsequently, all slices were stained with the nucleic acid probe Hoechst as described above.

After intensive washing and mounting on glass slides, all stained sections were dehydrated in a series of graded ethanol, processed through n-butylacetate and covered with Entellan.

\section{Confocal Microscopy}

Imaging of the fluorescence-labeled specimen was done using a confocal laser scanning microscope (LSM 510 Meta; Zeiss, Oberkochen, Germany). Excitation wave lengths were $633 \mathrm{~nm}$ (helium/neon2), $543 \mathrm{~nm}$ (helium/neon1), and $488 \mathrm{~nm}$ (argon). An ultraviolet laser (351/364 nm, Enterprise) was used to evoke the Hoechst fluorescence.

\section{Treatment Procedure}

According to the treatment procedure described in Heine et al. [12] (for a detailed time schedule, see fig. 3), the slice co-cultures were divided into different experimental groups and were treated with the following substances (final concentration): BAY60-7550 (10 $\mu \mathrm{M})$, ND7001 (10 $\mu \mathrm{M})$, MP-10 (10 $\mu \mathrm{M})$, and NGF $(50 \mathrm{ng} / \mathrm{ml})$. The experiments were performed in a blinded fashion, i.e. the compound identity was only provided after final data analysis.

The substances (except NGF) were dissolved in DMSO, sterilized by filters $(0.2 \mu \mathrm{m}$; Sarstedt, Nümbrecht, Germany) and finally added to $1 \mathrm{ml}$ incubation medium. To exclude influences resulting from the solvent DMSO (final concentration $0.1 \%$ ), an additional control group was used, treated only with ACSF (1\%). The substances were applied at each medium change (4 times within the incubation period).

\section{Tracing Procedure}

At DIV 8, small biocytin crystals (Sigma-Aldrich) of similar size were placed on the VTA/SN part of the co-cultures (according to [10]) under binocular control (for detailed time schedule, see fig. 3). Cultures were left in contact with the crystals for $2 \mathrm{~h}$ to allow the uptake of biocytin, followed by careful rinsing with fresh incubation medium. Then, the cultures were reincubated with medium containing the substances to allow for anterograde transport of the tracer. After DIV 10, the cultures were fixed as described above and cut into $50-\mu \mathrm{m}$ thick slices by means of the vibratome.

The visualization of the traced axons was performed using a previously described protocol [10]. Briefly, the anterogradely transported tracer biocytin was labeled using the ABC-Elite Kit (1:50; Vector Laboratories, Inc.), in combination with nickel/cobalt-intensified DAB. After mounting on glass slides, all stained sections were handled as described above. 


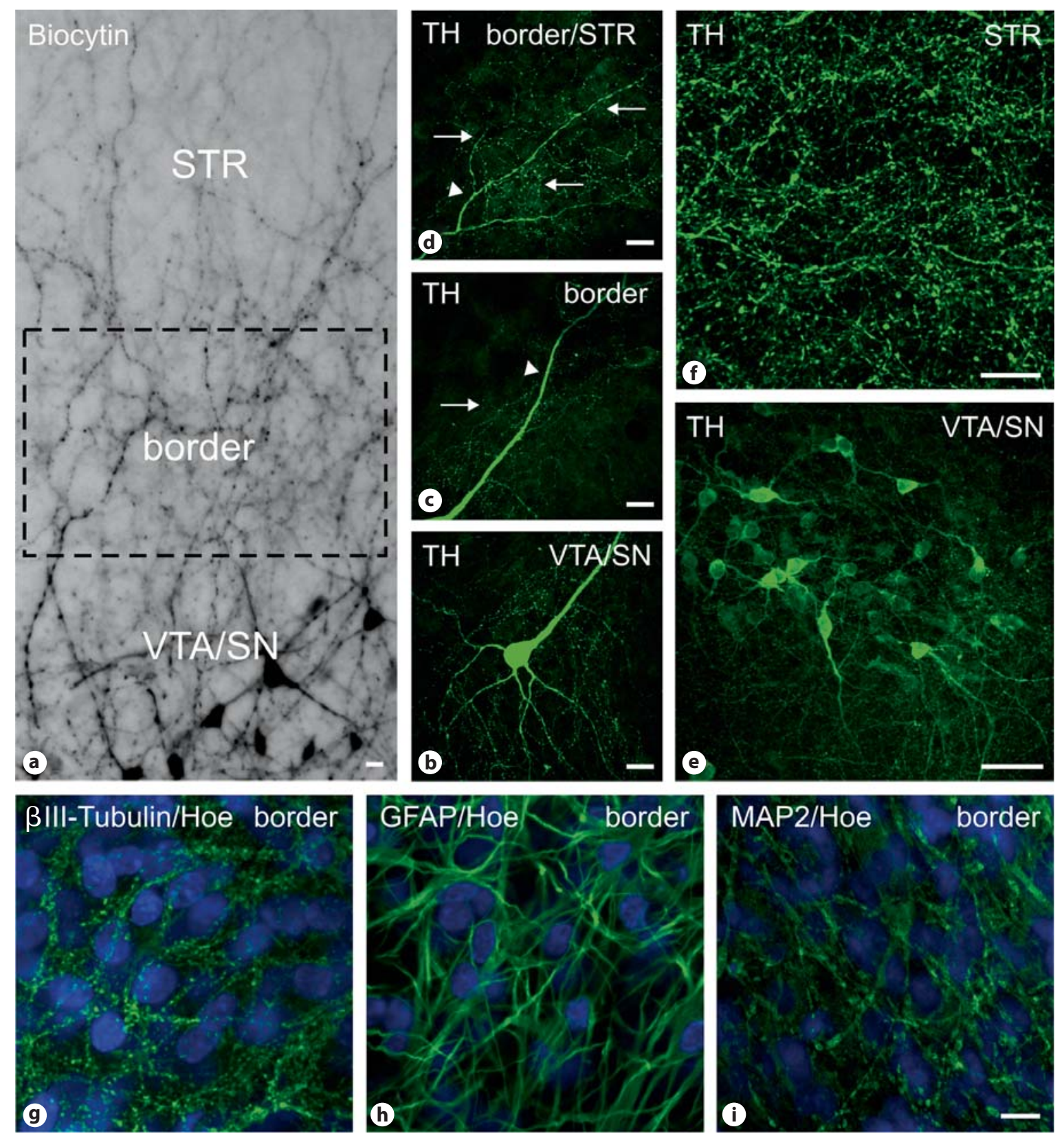

Fig. 4. a Overview of a DAergic nigrostriatal co-culture (VTA/ $\mathrm{SN}+\mathrm{STR}$ ) using the biocytin-tracing technique in combination with DAB labeling. The small biocytin crystals had been placed onto the VTA/SN part of the co-culture; afterwards, the anterograde tracer was transported from the cell bodies to the outgrowing fibers. The cell bodies (labeled black) of the VTA/SN and the respective outgrowing fibers, linking the border region and growing into the STR, are shown. b-f Examples of TH immunofluorescence labeling to characterize the expression of the DAergic marker in the co-cultures: TH-IR was observed on cell bodies and outgrowing fibers within the VTA/SN $(\mathbf{b}, \mathbf{e})$ as well as on fiber processes in the border region $(\mathbf{c}, \mathbf{d})$ and the STR $(\mathbf{d}, \mathbf{f})$. In the border region, strong TH-IR was observed (e.g. arrowhead in $\mathbf{c}, \mathbf{d}$ ) on fibers, but also an increased number of 'dot-like' structures (e.g. arrows in c, d) was found. Finally, within the STR, a fine network of TH-positive fibers and 'dot-like' structures is characteristic (f). g-i The expression of $\beta I I I-t u b u l i n-(\mathbf{g})$, GFAP- (astroglial marker) (h), as well as MAP2- (neuronal marker) (i) positive fibers in the border region is demonstrated, together with the nuclear stain Hoechst (Hoe) 33342. Scale bars: a $10 \mu \mathrm{m}$; b-d, f $20 \mu \mathrm{m}$; e $50 \mu \mathrm{m}$; g-i $10 \mu \mathrm{m}$. 


\section{Automated Image Quantification}

The quantification of outgrowing biocytin-traced fibers as well as of the TH-labeled fibers was performed within the border region of the co-cultures, i.e. the part where the two initially separated brain slices were grown together (fig. 4a). The following criteria were used for the selection of the biocytin-traced slices: (a) the tracer was correctly placed on the VTA/SN; (b) the major part of the VTA/SN was characterized by a dense network of biocytin-traced structures, and (c) no traced cell bodies had been observed in the target region STR (described in detail in [12]).

The raw images were obtained at $20 \times$ magnification from the whole border region by a usual transmitted light bright field microscope (Axioskop 50; Zeiss, Oberkochen, Germany) equipped with a CCD camera. Due to the usual problems with sensor noise and the large amount of light absorption and scattering inside the complex tissue structure of the stained co-culture slices, the axonal structures are typically blurred and image quality further suffers from inhomogeneous illumination. The border region is composed of both vesicular and fibrous structures (in the following only termed fibers) due to the dynamic transport properties of the tracer biocytin. Thus, a tailored image analysis pipeline had to be designed to quantify the fiber density in an automated manner. This procedure can be roughly split into two parts: image pre-processing and fiber detection.

\section{Pre-Processing of the Images}

At first, a deconvolution of the images was computed to reduce the blur and highlight the expected 'true' structures of the fibers. Since the real point-spread function of the microscope setup was lacking, the convolution kernel was estimated by a general Gaussian function (the width of the Gaussian is empirically set to 2 pixels), which is a reasonable guess in the general case of isotropic blur. The actual deconvolution was done with a damped least squares method (see [27]).

The deconvolution step inevitably leads to an increase in noise. Since noisy image structures would lead to a high number of false positive hits for subsequent detection of vesicular structures, a total variation-based smoothing step was applied to suppress single pixel noise in the images while preserving structures of interest [28].

Large-scale blurry structures in the background (due to structures from out-of-focus structures in the tissue) were subsequently removed by applying a top-hat transform, image structures larger than a given threshold were removed (a cutoff of 40 pixels was used here, see [29] for details).

\section{Quantification of the Fiber Density}

After pre-processing, the fiber structures appeared as well-defined bright regions that clearly stood out against the background. These regions were then extracted by Otsu thresholding [30].

Subsequently, the image area occupied by the fibers was measured to obtain a reasonable estimate of the underlying axonal density of the analyzed specimen. Precisely, the ratio of the number of foreground pixels (fibers) against the total number of pixels in the images was taken, giving the percentage of area occupied by fibers in the focal plane.

\section{Statistics}

Statistical analysis of the intracellular cGMP assay was performed using Student's t test. Statistically significant differences were considered at a p level $<0.05\left({ }^{*} \mathrm{p}<0.05,{ }^{* *} \mathrm{p}<0.01,{ }^{* *} \mathrm{p}<\right.$ 0.001; the error bars indicate the SEM).

Statistical analysis of the quantitative data, comparing the different treatment groups, was performed using a Wilcoxon Rank Sum test. Each individual group was compared to the control group DMSO. To correct for multiple pairwise testing between groups, a conservative Bonferroni p value correction was applied. Statistically significant differences were considered at a p level $<0.05\left({ }^{*} \mathrm{p}<0.05,{ }^{* *} \mathrm{p}<0.01,{ }^{* * *} \mathrm{p}<0.001\right)$. For each group, the treatment experiments were repeated for at least three individual preparations.

\section{Results}

\section{$I C_{50}$ Values of Selected PDE-Is}

The potency and selectivity of BAY60-7550, ND7001, and MP-10 were confirmed against individual human purified proteins of each PDE family (except for PDE6, which was isolated from bovine retina), and the results are shown in table 1.

The data of the $\mathrm{IC}_{50}$ values against $\mathrm{PDE} 2 \mathrm{~A}$ indicated the following ranking: BAY60-7550 > ND7001 > MP-10. The PDE2-I BAY60-7550 inhibited the target enzyme with high potency with an $\mathrm{IC}_{50}$ value of $0.18 \mathrm{nM}$, and ND7001 was characterized as a PDE2-I with moderate affinity $\left(\mathrm{IC}_{50}=57 \mathrm{nM}\right)$. Both compounds are highly selective against all other PDE isoforms. MP-10 inhibited the activity of PDE10 with high potency $\left(\mathrm{IC}_{50}=1.14 \mathrm{nM}\right)$ as well as high selectivity towards each individual isoform of the PDE family. In general, PDE proteins show a high degree of sequence homology across different species, especially within their catalytic domains (for review, see $[17,18])$. Thus, it is very unlikely that PDE-Is show species-specific effects with regard to their inhibitory activity. We have tested, for example, the PDE2-I ND7001 in a reference experiment using rat and human PDE2 proteins and could not detect any significant differences in their inhibitory activity across species (data not shown).

\section{Effects of PDE2-Is on Intracellular cGMP Level}

The PDE2-Is BAY60-7550 and ND7001 had been selected for the cellular cGMP assay. Measurement of cGMP levels in HEK293 cells, stably transfected with the full-length sequence of human PDE2A, confirmed that PDE2-I BAY60-7550 (fig. 5a) and ND7001 (fig. 5b) increased cGMP levels in a dose-dependent manner. In the presence of SNP $(100 \mu \mathrm{M})$, these compounds significantly increased cGMP levels as compared to the control group. These findings suggest that PDE2 may play a major role in the degradation of cGMP under conditions of 


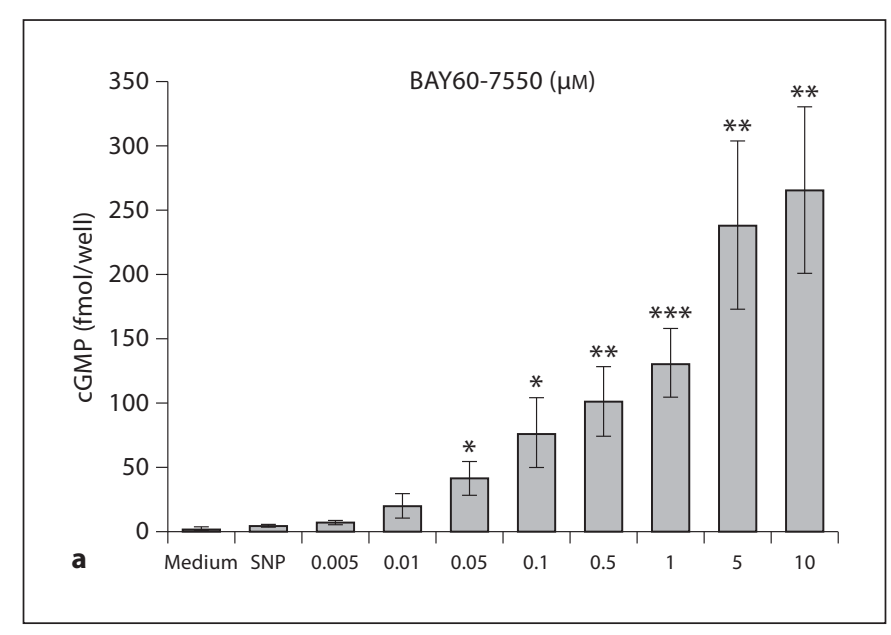

Fig. 5. Dose-dependent effects of PDE2-Is BAY60-7550 (a) and ND7001 (b) on intracellular cGMP levels in a PDE2-HEK293 cell line. BAY60-7550 and ND7001 (in the presence of the GC stimulator SNP) increased the intracellular cGMP level in a dose-dependent fashion. Values are shown in fmol cGMP per well. The

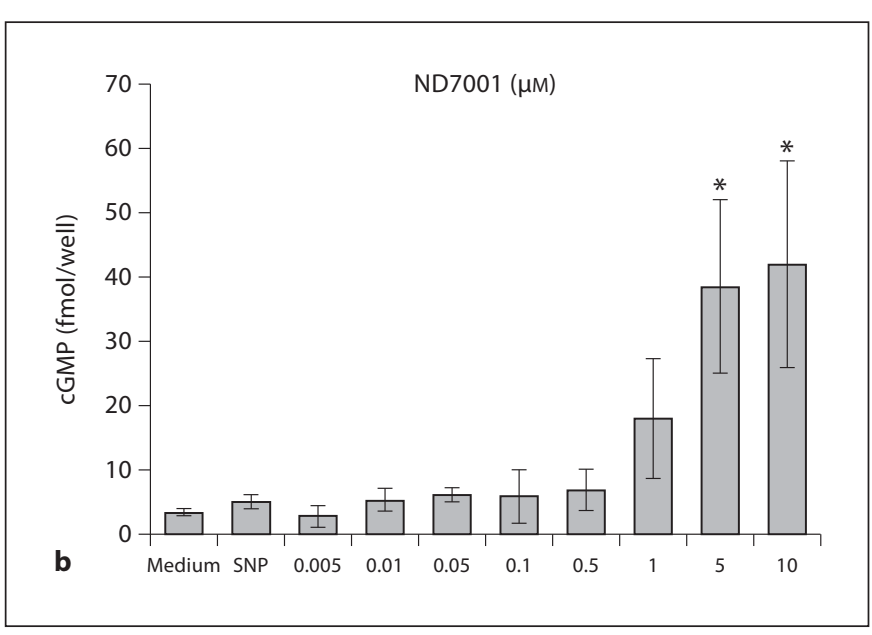

error bars indicate SEM. The numbers of independent experiments per compound were: BAY60-7550 $(\mathrm{n}=8)$ and ND7001 $(\mathrm{n}=$ 4 ), performed in duplicates. Differences to SNP were considered to be statistically significant according to a p level $<0.05$ ( ${ }^{*} \mathrm{p}<$ $\left.0.05,{ }^{* *} \mathrm{p}<0.01,{ }^{* *} \mathrm{p}<0.001\right)$.
GC stimulations. The numbers of independent experiments per compound were: BAY60-7550, $\mathrm{n}=8$, and ND7001, $\mathrm{n}=4$, performed in duplicates.

\section{Characterization of the Organotypic Co-Cultures (VTA/SN+STR)}

In the present study, the VTA/SN and the STR, main parts of the DAergic nigrostriatal projection system, were prepared from P1-5 old rats, a period during which the rat exhibits an adult localization of the neurons, although the axonal network is still immature [31]. Following fixation of the cultures at DIV 10, the slices were characterized using different labeling techniques.

\section{Biocytin Tracing}

The morphology of the biocytin-traced cell bodies as well as the outgrowth of the fibers were analyzed in treated as well as untreated control co-cultures. An example of an untreated biocytin-traced co-culture is shown in figure $4 \mathrm{a}$. In general, numerous biocytin-marked fibers (labeled in black) originating from the VTA/SN, ramified and crossed the border region between the two brain slices. Moreover, their fiber processes were observed to grow into the striatal part of the culture. The fiber pathways of the VTA/SN+STR co-cultures developed an innervation pattern similar to that found in vivo [10]. These findings are in accordance with other studies, showing that the projections of axons to their targets follow special stereo- typical routes during the development of the central nervous system and that selected guidance molecules support this innervation [32-34].

\section{Immunohistochemistry/Immunofluorescence}

The expression of $\mathrm{TH}$, a marker for DAergic neurons, was shown using immunofluorescence labeling in combination with confocal laser scanning microscopy. The results indicate the presence of $\mathrm{TH}$-positive cell bodies and fibers in the VTA/SN (fig. 4b, e), whereas the expression of the marker was restricted to fibers within the border region (fig. 4c, d) and the STR (fig. 4d, f). Fluorescence images illustrating the differences in the expression of TH immunoreactivity (IR) in the respective anatomical parts of the DAergic system are shown in figure $4 \mathrm{~b}-\mathrm{d}$. The pictures especially exemplify the expression of the 'dot-like' structures in the border region and the target area (also observed after biocytin tracing, see fig. 6). Furthermore, antibodies against neuronal markers, i.e. $\beta I I I-t u b u l i n$ and MAP2 and the astroglial marker GFAP, were used to prove the existence of neuronal processes and glial cells, especially within the border region of the co-cultures. A positive IR for all investigated markers was observed in VTA/SN and STR. Examples are shown in figure $4 \mathrm{~g}-\mathrm{i}$, where the existence of $\beta$ III-tubulin- (fig. 4g), MAP2(fig. 4i), and GFAP- (fig. 4h) labeled fibers spanning the border region and, thus, linking the two slices could be verified. These results are consistent with the results 

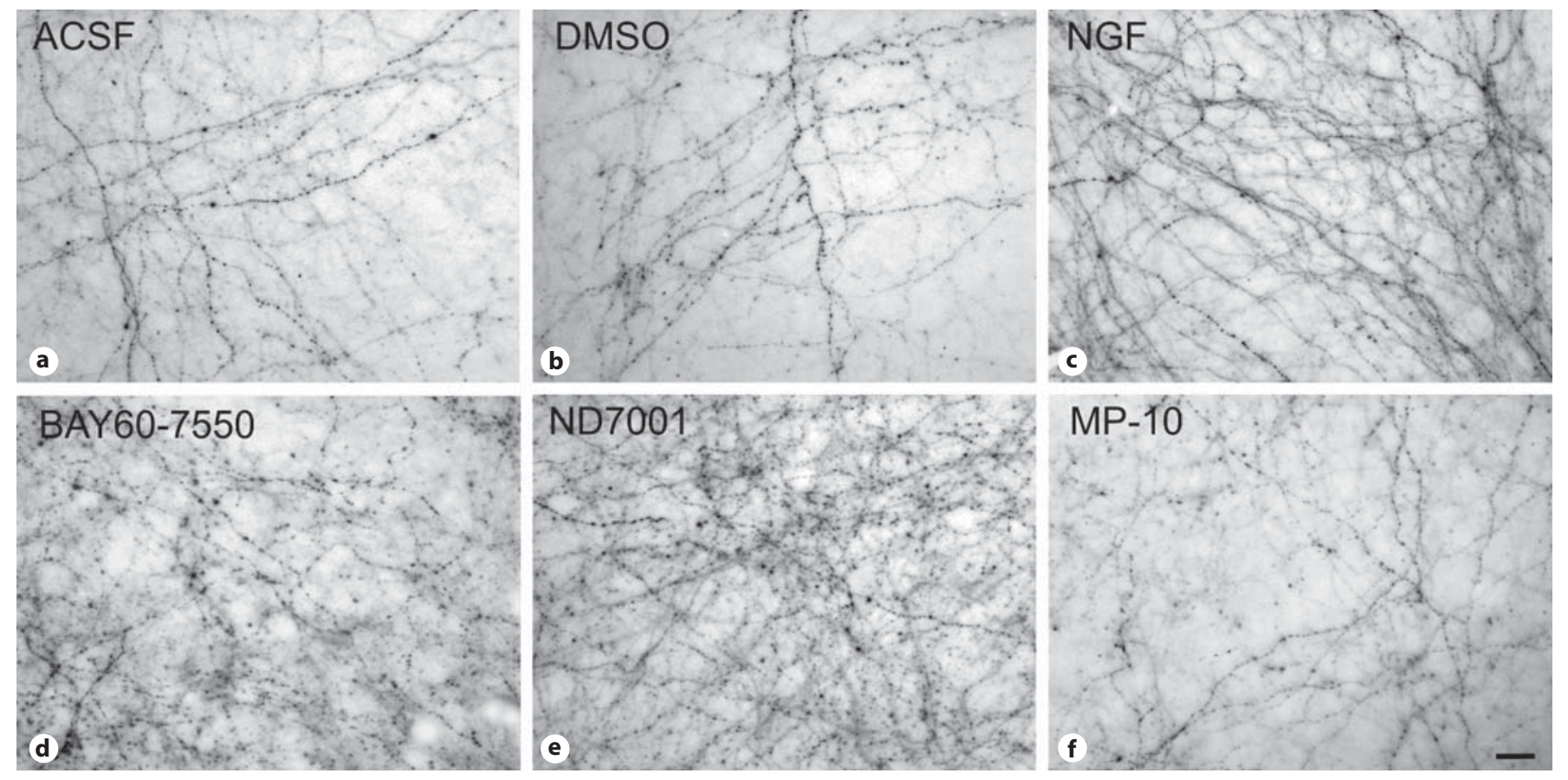

Fig. 6. Results of the biocytin tracing after substance treatments are demonstrated within the border region of the co-cultures. Compared to control conditions applying ACSF (a) and DMSO (b) an increase in biocytin-positive fibers in PDE2-Is-treated cocultures using BAY60-7550 (d) and ND7001 (e) was observed.
These effects were comparable with the effect evoked by the growth factor NGF (c). Co-cultures treated with MP-10 (f) were characterized by few biocytin-traced innervations within the border region. Scale bar: a-f $20 \mu \mathrm{m}$. found in our previous studies, demonstrating the expression of neuronal and glial fiber connections within VTA/ $\mathrm{SN}+$ prefrontal cortex co-cultures [12].

Further light microscopic and triple immunofluorescence labeling studies with specific antibodies directed against PDE2A and PDE10 were performed to demonstrate the expression of these isoforms. Examples are shown in figure 7.

The light microscopic data indicated the expression of PDE2A on cell bodies and fibrous structures in the STR and VTA/SN (data not shown) and correlate with the immunofluorescence data. The immunofluorescence labeling was predominately found at the plasma membrane as well as in the cytoplasm (an example for the STR is given in fig. 7a-c, arrows). Moreover the co-localization of the PDE2A-IR on MAP2-immunopositive cells could be observed (example for VTA/SN; fig. 7f-h, arrows). We did not find PDE2-positive fibers in the border region (an example is shown in fig. 7d, e). Double immunofluorescence experiments using $\mathrm{TH}$ and $\mathrm{PDE} 2 \mathrm{~A}$ indicated no co-expression on cell bodies or fibers in all parts of the studied co-cultures, as shown in figure $7 \mathrm{i}-\mathrm{m}$.

Phosphodiesterase 2 Inhibitors Promote Axonal Outgrowth
Light microscopic images also confirmed the evidence of PDE10-marked cell bodies and fibers in the STR and the VTA/SN. Additional investigations using double immunofluorescence labeling with antibodies against TH and the PDE10 isoform indicated the expression of PDE10 on cell bodies and single fibers. On the other hand, no co-expression with TH-positive fibers (STR, VTA/SN) or the localization on TH-positive neurons (VTA/SN) could be found (fig. $7 \mathrm{n}-\mathrm{q}$ ).

The obtained results thus clearly underline the expression of the investigated isoforms in the VTA/SN+STR cocultures during development.

\section{Treatment of Organotypic Co-Cultures with PDE2-} and PDE10-Is

Qualitative Results of the Fiber Growth (Biocytin Tracing)

Further co-cultures (VTA/SN+STR) had been used to study the influence of selected PDE-Is, with different subclass specificities, on fiber growth and sprouting. All experiments were performed in a blinded fashion, i.e. the compound identity was provided after final data analysis. 

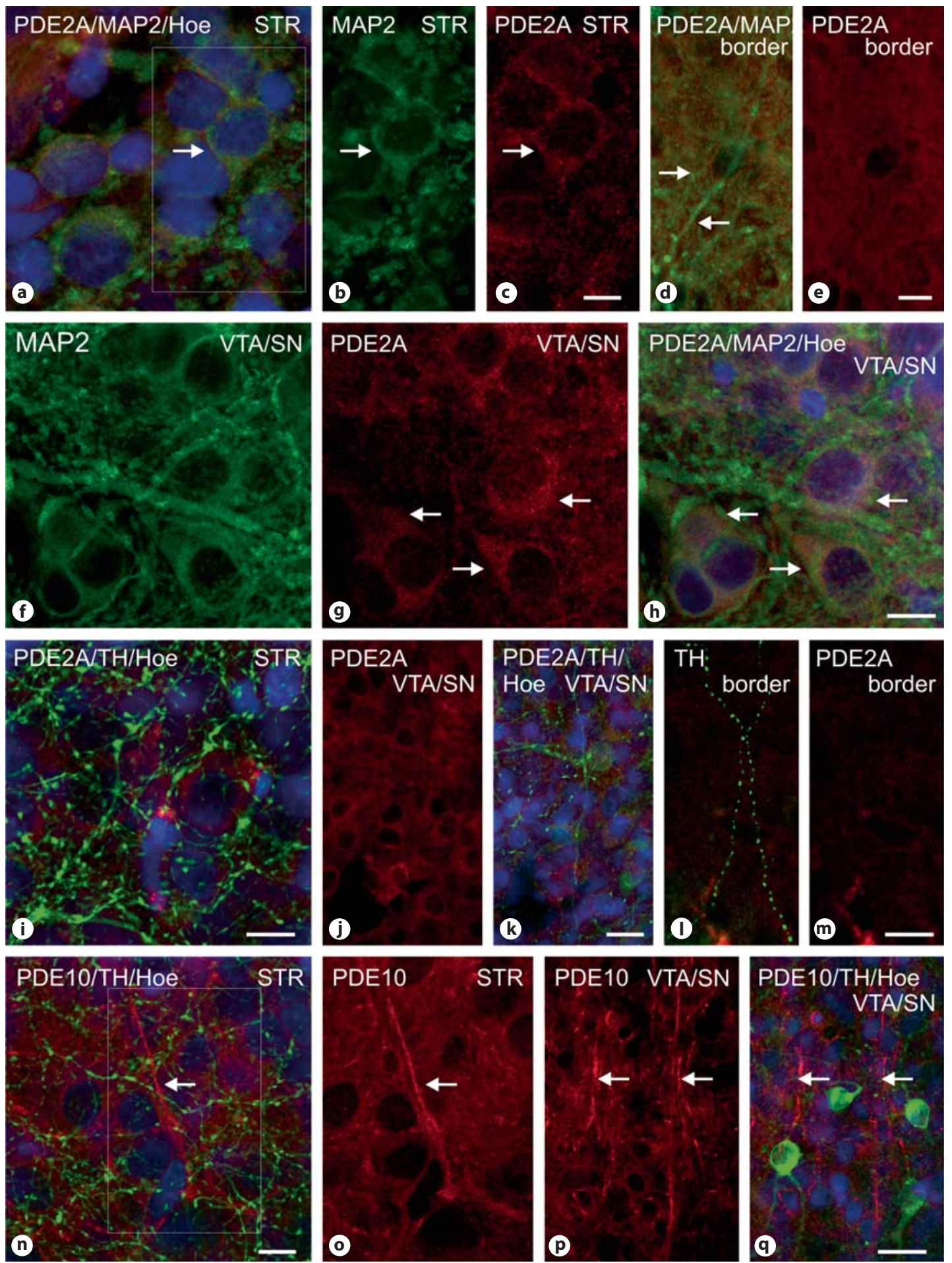

Fig. 7. Confocal images of multiple immunofluorescence labeling using antibodies against PDE2A (a-m) and PDE10 (n-q) in combination with MAP2 (neuronal marker), TH (DAergic marker), and Hoechst (Hoe) are shown. $\mathbf{a}-\mathbf{h}$ PDE2A is expressed in the STR and VTA/SN especially on cell bodies (plasma membrane, cytoplasm; co-expression with MAP2 is indicated by arrows). No labeling was found on fibers in the border region (example given in

d, e). The double-labeling experiments with antibodies against $\mathrm{TH}$ indicated neither co-localization of PDEA2 and TH in the STR, VTA/SN, nor in the border region (i-m). PDE10 was found to be expressed on cell bodies and also on fibers (indicated by arrows in $\mathbf{n - q}$ ), but no co-localization was found with TH-positive structures in the studied regions. Scale bars: a-c $5 \mu \mathrm{m}$; $\mathbf{d}-\mathbf{h} 10$ $\mu \mathrm{m} ; \mathbf{i} 20 \mu \mathrm{m} ; \mathbf{j}, \mathbf{k} 20 \mu \mathrm{m} ; \mathbf{l}-\mathbf{o} 10 \mu \mathrm{m} ; \mathbf{p}, \mathbf{q} 20 \mu \mathrm{m}$. 

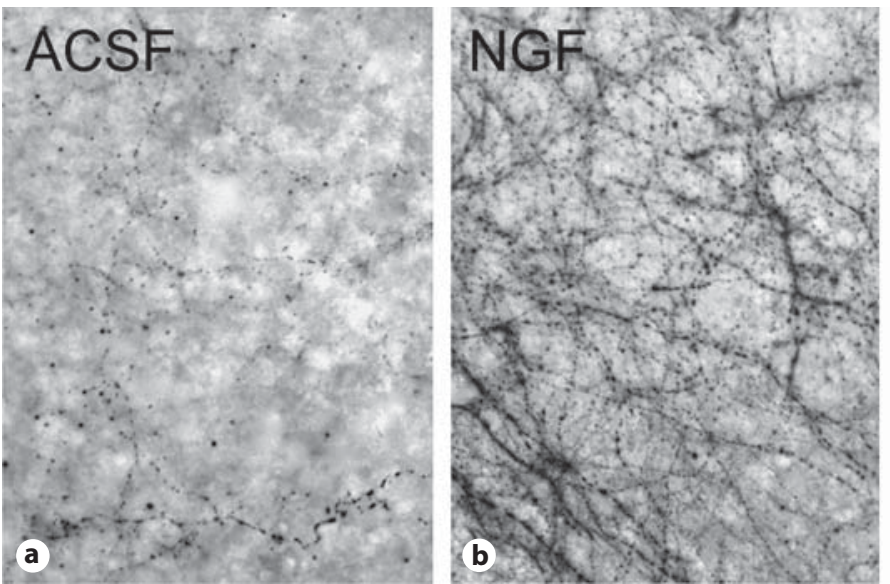

Fig. 8. a-d TH-positive fibers after substance treatment (a ACSF; b NGF, c DMSO, d BAY60-7550 solved in DMSO) within the border region of the co-cultures are shown (scale bar: $\mathbf{a}-\mathbf{d} 20 \mu \mathrm{m}$ ). e Results of automated image quantification to measure the density of the TH-positive fibers in the border region of differently treated co-cultures in comparison to control conditions. After $\mathrm{TH}$ labeling, the fiber density was quantified, taking the ratio of the number of foreground pixels (fibers) over the total number of pixels in the images to reveal the size of the area occupied by fibers. The box-whisker plots represent the empirical distribution of the fiber densities in the different groups (the vertical axis corresponds to the percentage of image pixels belonging to labeled fibers). Compared with ACSF and DMSO, the growth factor NGF induced a significant increase in TH-positive fibers in the studied co-cultures. In contrast, the PDE2-I BAY60-7550 evoked only a small but significant increase of the number of TH-positive fibers, in comparison with the solvent DMSO. The numbers of analyzed images per group are: ACSF $(n=8)$, DMSO $(n=20), \operatorname{NGF}(n=33)$ and BAY60-7550 ( $\mathrm{n}=94)$. Differences were considered statistically significant at a p level $<0.05\left({ }^{*} \mathrm{p}<0.05,{ }^{* *} \mathrm{p}<0.001\right)$.

As described in the Methods section, the co-cultures were treated 4 times with the respective substances, over an incubation time of 10 days and, afterwards, the outgrowth of the biocytin-traced fibers in the border region was analyzed.

Differences in fiber outgrowth within the border region could be observed for the different treatments, and examples of these results are illustrated in figure 6. An increase in biocytin-positive fibers was apparent in PDE2-I- (e.g. BAY60-7750, fig. 6d) treated co-cultures in comparison to control conditions (ACSF, fig. 6a; DMSO, fig. 6b). The growth factor NGF evoked an effect comparable to the PDE2-Is under study (fig. 6c). Co-cultures treated with MP-10, an inhibitor of the PDE10, were characterized by fewer biocytin-traced innervations within the border region compared to the effects induced by the PDE2-Is (fig. 6f). Furthermore, the 'dot-like' structures
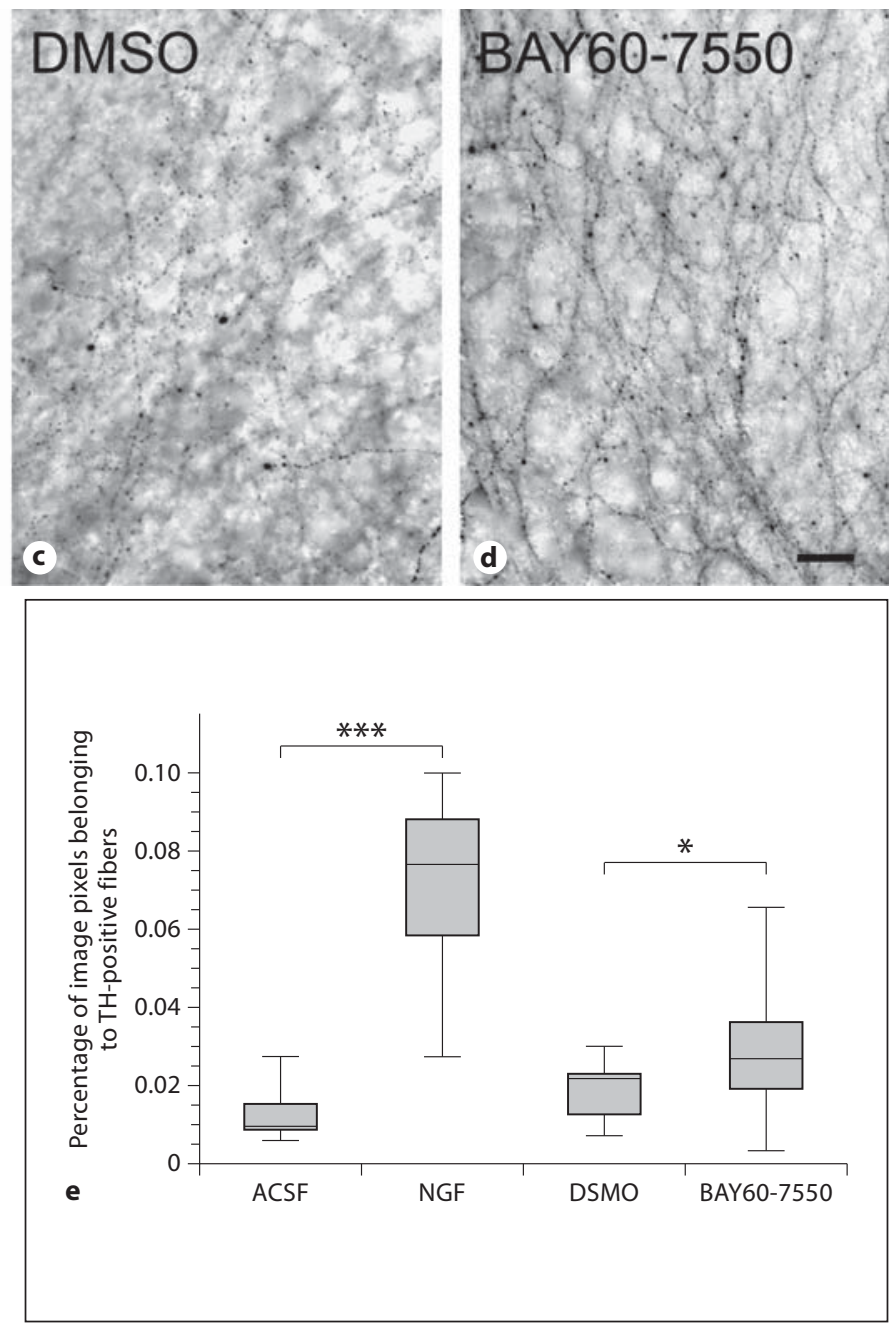

are of particular interest. They could be observed in the border region and the target region (STR) after substance treatment. This expression was also found after TH labeling of outgrowing fibers (see also fig. 4, 8). A significant increase in the number of puncta was noticeable after treatment with BAY60-7550.

Quantification of Fiber Density (Biocytin Tracing)

Starting from light microscopic images, the density of the biocytin-positive fibers was measured for all treatment groups in the border region of the co-cultures using the automated image quantification described above. The respective results of the tested substances are shown as box-whisker plots in figure 9. A significant increase in neuronal fiber outgrowth after application of the PDE2Is BAY60-7550 and ND7001 was observed, with BAY607550 exhibiting the strongest effect. These results were of 


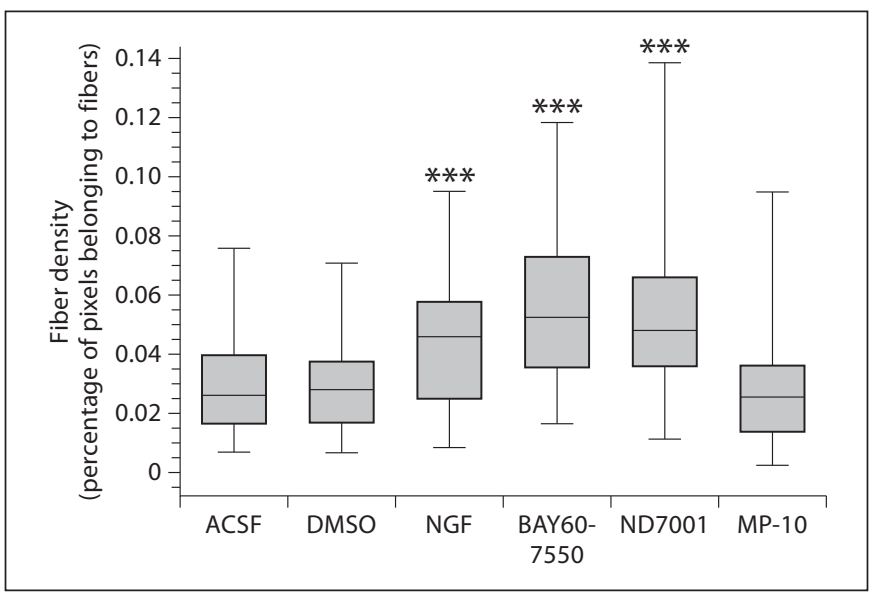

Fig. 9. Automated image quantification to measure the fiber density in the border region of different treated co-cultures in comparison to control conditions. After biocytin tracing, the fiber density was quantified, taking the ratio of the number of foreground pixels (fibers) over the total number of pixels in the images to reveal the size of the area occupied by fibers. The boxwhisker plots represent the empirical distribution of the fiber densities in the different groups (the vertical axis corresponds to the percentage of image pixels belonging to traced fibers). The analysis revealed a significant increase in neuronal fiber outgrowth after application of PDE2-Is BAY60-7550 and ND7001, with effects comparable to the stimulation capacity of NGF. The inhibitor of PDE10, MP-10, caused a significantly lower fiber density in the border region, similar to the results of ACSF and DMSO. The numbers of analyzed images per group were: $\operatorname{ACSF}(n=22)$, DMSO $(\mathrm{n}=62)$, NGF $(\mathrm{n}=41)$, BAY60-7550 ( $=101)$, ND7001 $(\mathrm{n}=103)$, and MP-10 $(\mathrm{n}=76)$. Statistically significant differences were considered at a p level $<0.05\left({ }^{* * *} \mathrm{p}<0.001\right)$.

comparable effect size as for the neurotrophic factor NGF. MP-10 (PDE10-I) caused a clearly lower fiber density in the border region, akin to the effects observed in the control groups. Moreover, no significant differences in fiber density could be observed between the ACSF and the DMSO control group, thus excluding adverse effects resulting from the solvent DMSO. The numbers of analyzed images per group are: ACSF $(\mathrm{n}=22), \operatorname{DMSO}(\mathrm{n}=$ $62)$, NGF $(\mathrm{n}=41)$, BAY60-7550 $(\mathrm{n}=101), \mathrm{ND} 7001(\mathrm{n}=$ 103), and MP-10 ( $\mathrm{n}=76)$.

Treatment of Organotypic Co-Cultures with PDE2-I and NGF - Characterization of DAergic Fibers

Qualitative Results of the Fiber Growth (TH

Immunolabeling)

Treatment of the co-cultures with ACSF, NGF, DMSO, and BAY60-7550 (solved in DMSO) and immunolabeling with antibodies against $\mathrm{TH}$ indicated differences in the expression of TH-positive fibers in the border region (examples are shown in fig. 8a-d). Especially the effect of NGF as trophic factor was clearly visible.

\section{Quantification of the Fiber Density}

(TH Immunolabeling)

Moreover, the density of the TH-positive fibers was analyzed and quantified for all treatment groups as described above. The results are shown in figure $8 \mathrm{e}$. The growth factor NGF induced a significant increase in $\mathrm{TH}$ positive fibers in the studied co-cultures as compared to ACSF and DMSO. In contrast, the PDE2-I BAY60-7550 evoked a smaller (in comparison to the biocytin tracing) but statistically significant increase in the density of THpositive fibers under these conditions (as compared with the DMSO group). The observed difference in effect size of BAY60-7550 treatment between biocytin tracing and TH labeling suggests an involvement of additional neurotransmitter populations, other than the TH-positive (DAergic) subgroup. Although small differences between DMSO and ACSF exist, they were not found to be statistically significant.

The numbers of analyzed images per group are: ACSF $(\mathrm{n}=8)$, DMSO $(\mathrm{n}=20)$, NGF $(\mathrm{n}=33)$, and BAY60-7550 $(\mathrm{n}=94)$. Differences were considered statistically significant at a p level $<0.05\left({ }^{*} \mathrm{p}<0.05,{ }^{* *} \mathrm{p}<0.01,{ }^{* *} \mathrm{p}<\right.$ $0.001)$.

\section{Discussion}

The characterization of determinants of innervation patterns and the activities of growth promoting factors are of special interest for the development of clinical strategies to promote fiber development and, thereby, the regeneration of neuronal circuits. Using the ex vivo model of organotypic slice co-cultures, consisting of the VTA/ SN and the STR, in combination with biocytin tracing, $\mathrm{TH}$ immunolabeling, and subsequent automated image quantification, it was shown that substances with the potential to inhibit PDE2 (BAY60-7550, ND7001) were able to increase neuronal fiber outgrowth, in contrast to the studied PDE10-I (MP-10) and the control substances ACSF and DMSO.

The data suggest a role for PDE2-Is, possibly mediated via cGMP, in the induction of fiber outgrowth in the nigrostriatal projection system. In PDE2-transfected HEK293 cells, a dose-dependent increase of intracellular cGMP levels in response to PDE2-Is, the previously described reference compounds BAY60-7550 and ND7001, 
could further be shown. These data indicate that inhibition of PDE2 results in a dose-dependent augmentation of intracellular cGMP levels.

In vivo studies on rats have shown that DAergic neurons emerged around embryonic day 10, with the first nerve fibers appearing in the STR around embryonic days 14-15. The innervations are completed at about P15 [35]. Thus, the situation in the established ex vivo model strongly correlates with the developmental processes of physiological neuronal circuits. The observed neurite outgrowth pattern is consistent with our own previous data obtained under in vivo conditions [10].

PDEs are involved in distinct tasks in the brain, both in the mature rat brain and at embryonic as well as postnatal stages when significant developmental and maturational events are in progress $[19,20,36]$. An increase in PDE activity was reported during development of the brain from fetus to adulthood $[37,38]$. Our data indicate an expression of PDE2A and PDE10 on cells in the DAergic co-culture preparations, consequently underlining a role of these isoenzymes in early development.

Hydrolyzing PDE families have distinct expression patterns within the central nervous system. For instance, striatal medium spiny neurons express mRNA for the $\mathrm{PDE} 1 \mathrm{~B}, 2 \mathrm{~A}, 4 \mathrm{~B}, 7 \mathrm{~B}, 9 \mathrm{~A}$, and 10A isoforms (for references, see $[39,40])$. A prominent PDE2A-IR was found besides the STR in the isocortex, hippocampus, and basal ganglia. Moreover, a high expression was observed in the SN and VTA on cell bodies and terminals [20,36], which is supported by the findings presented in this work. PDE10 mRNA and protein are highly enriched in medium spiny neurons in the STR, within the cell bodies and dendrites as well as on SN axons [22, 41, 42]. PDE10A is associated with post-synaptic membranes of these medium spiny neurons and their dendrites and spines [43].

Under physiological conditions, the dual substrate enzyme PDE2(A) plays a critical role in the feedback control of cellular cAMP and cGMP levels, resulting in acute and long-term changes of neuronal functions. The basal PDE2 activity in neurons is low, but it is stimulated by an acute increase in cGMP following GC activation during signal transduction. In detail, a characteristic feature of PDE2 is the positive cooperativity with the substrate cGMP due to an allosteric cGMP binding site at N-terminal domains $[40,44]$. This cyclic nucleotide stimulates cAMP degradation such that higher concentrations of cGMP inhibit cAMP hydrolysis and low levels of cGMP enhance the rate of cAMP hydrolysis $[39,44]$. Besides the described features of PDE2, also special GAF domains of PDEs 5, 6, 10, and 11 bind cGMP and may regulate the catalytic activity

Phosphodiesterase 2 Inhibitors Promote

Axonal Outgrowth or protein-protein interactions (for references, see [45]). As a consequence, inhibition of PDE2 selectively increases cGMP and cAMP in brain active synapses [19, 44]. Incubation of cultured neurons and hippocampal slices with the selective PDE2-I BAY60-7550 resulted in increased cGMP levels [44]. This is in accordance with our present data, indicating a dose-dependent increase of intracellular cGMP levels in response to the investigated PDE2-Is obtained in the HEK cell line. Inhibition of the PDE10A causes also increased levels of cAMP and cGMP [40], with the difference that the regulation of the two cyclic nucleotides by PDE10A is independent [22].

The involvement of cAMP/cGMP during neuronal development in synaptic plasticity and memory formation has been described [19, 36, 44]. Recent data suggest a role of cAMP (via cAMP-dependent protein kinase) in neuritogenesis and synaptogenesis during neuronal differentiation in NG108-15 cells [14]. The particular role of exchange proteins directly activated by cAMP (Epac) will be of specific interest for further studies as they are known to be involved in mediating cAMP responses. These are implicated in various cellular processes such as integrin-mediated cell adhesion and cell-cell junction formation or influencing, for example, cortical actin cytoskeleton (for review, see [46]).

cGMP can regulate directional guidance of growth cones $[13,47]$ and is involved in the outgrowth of cortical neurons during maturation [32]. The observed trophic effects clearly show that inhibitors of PDE2 are able to modulate neuronal fiber outgrowth in VTA/SN+STR cocultures in contrast to the studied PDE10-I (MP-10), suggesting an involvement of PDE2, via cGMP signaling, in synaptic plasticity. Data from Boess et al. [44] confirm that inhibition of PDE2, e.g. by the selective PDE2-I BAY60-7550, increases cGMP/cAMP in cultured neurons and hippocampal slices, resulting in enhanced long-term potentiation of synaptic transmission, which is thought to be a key factor implicated in pro-cognitive activity without affecting basal synaptic transmission. A role of PDE2A activity in memory processes is further supported by the results of Song et al. [47] and Rutten et al. [19] showing that BAY60-7550 reportedly improves performance of rodents in recognition memory tasks.

Inhibition of PDE10 promotes effects modulating basal ganglia function in ways that suggest a particular therapeutic utility in the treatment of psychosis in schizophrenia $[22,23,48]$. It has also been shown that PDE10-I increases phosphorylation of key cAMP-dependent substrates, such as CAMP response element-binding protein, extracellular receptor kinase, or, more recently, the 
AMPA-receptor GluR1 subunit [48-51]. Sotty et al. [51] further suggest a modulatory role of PDE10A on mesolimbic DAergic neurotransmission, via the D1-regulated feedback loop controlling midbrain DAergic neuronal activity. Therefore, investigating its modulatory role to affect the confluence of the corticostriatal glutamatergic and the midbrain DAergic pathways will be of particular interest.

The regulation of cAMP synthesis by DA and other neurotransmitter receptors has been extensively studied. But the importance of cAMP degradation by PDEs and the precise roles of each PDE isoform in DAergic signaling are not yet completely understood, owing to the diversity of PDE isoforms expressed in the STR and the complexity of their potential regulation (for review, see [24]). The authors conclude that the inhibition of PDEs upregulates $\mathrm{CAMP} / \mathrm{PKA}$ signaling in three neuronal subtypes, resulting in (a) the stimulation of DA synthesis at DAergic terminals, (b) the inhibition of D2 receptor signaling in striatopallidal neurons, and (c) the stimulation of D1 receptor signaling in striatonigral neurons. However, it should be noted that the striatopallidal system as well as the corticostriatal glutamatergic projection are not constituents of the organotypic slice co-culture system used in the present study - possibly causing the different inhibitory effects of the investigated PDE-Is (especially for PDE10-I). However, the mechanism of inhibition responsible for the observed effects of the two kinds of PDE-Is needs further experimental investigations, especially the role of PDE10 to affect the corticostriatal glutamatergic and the midbrain DAergic pathways.

Finally, in the present study, the growth-promoting effect of the PDE-Is was compared with the stimulating capacity of NGF, which elicited a significant increase in fiber outgrowth intensity. Neurotrophins, like NGFs and their receptors, are the major regulators of neuronal survival during development, after injury or nervous system diseases [52]. NGF promotes neurite extension through a cAMP-independent signaling pathway involving Ras, $\mathrm{PKC}$, and extracellular signal-regulated protein kinase [53]. It has been shown that cAMP also induces neuronal differentiation in PC12 cells and that the co-treatment of NGF and dibutyryl cAMP exhibits synergistic effects on neurite outgrowth [54]. Moreover, NGF increases cGMP level and activates PDEs in PC12 cells [55]. Also other trophic molecules have been shown to enhance survival and neurite outgrowth of mesencephalic DAergic neurons in single cell cultures or slice cultures [56-58]. Treatment of organotypic slice co-cultures with BDNF resulted in enhanced survival of TH-positive neurons and an increased growth of $\mathrm{TH}$-positive fibers into the striatal tissue [58]. GDNF has been shown to act as a powerful chemoattractant for outgrowing DAergic axons in organotypic co-cultures $[33,59]$ as well as under in vivo conditions [60]. Finally, Thompson et al. [60] showed that axonal regrowth in the nigrostriatal trajectory can be further enhanced by the addition of trophic support by overexpression of GDNF in the striatal target.

In summary, cAMP/cGMP are involved in survival, repair, and remodeling in the nervous system both during development and after injury, and PDE2 appears to act as an important regulator in these processes. In the present study, we were able to analyze the specific potential of PDE-Is in mediating the growth of defined fiber connections under controlled conditions, using the ex vivo model of organotypic slice co-cultures of the nigrostriatal system in combination with an automated image quantification. Hence, our results strongly support a trophic role of PDE2-Is in shaping interneuronal connections during the early phase of neuronal development. The characterization of the molecular and functional basis underlying the observed effects of PDE2-Is in regeneration and repair of disrupted neuronal circuits will be an important next step to foster the development of improved ways for the treatment of neurological disorders.

\section{Acknowledgements}

The authors thank Katrin Becker and Katrin Krause (Rudolf Boehm Institute of Pharmacology and Toxicology) for technical assistance.

The work presented in this paper was made possible by funding from the German Federal Ministry of Education and Research (BMBF, PtJ-Bio, 0313909) and was supported by the European Fund for Regional Development (EFRE) and by the Free State of Saxony (grant No. SAB12525).

References

1 Redgrave P, Rodriguez M, Smith Y, Rodriguez-Oroz MC, Lehericy S, Bergman H, et al: Goal-directed and habitual control in the basal ganglia: implications for Parkinson's disease. Nat Rev Neurosci 2010;11:760-772.

-2 Sesack SR, Carr DB: Selective prefrontal cortex inputs to dopamine cells: implications for schizophrenia. Physiol Behav 2002;77:513517.

-3 Dailly E, Chenu F, Renard CE, Bourin M Dopamine, depression and antidepressants. Fundam Clin Pharmacol 2004;18:601-607.

4 Wise RA: Roles for nigrostriatal - not just mesocorticolimbic - dopamine in reward and addiction. Trends Neurosci 2009;32: 517-524.

Heine/Sygnecka/Scherf/Berndt/ Egerland/Hage/Franke 
5 Hökfelt T, Johansson O, Fuxe K, Goldstein M, Park D: Immunohistochemical studies on the localization and distribution of monoamine neuron systems in the rat brain. I. Tyrosine hydroxylase in the mes- and diencephalon. Med Biol 1976;54:427-453.

6 Fallon JH, Loughlin SE: Substantia nigra; in Paxinos G (ed): The Rat Nervous System. San Diego, Academic Press, 1995, pp 215-237.

7 Lindvall O, Björklund A: Cell therapy in Parkinson's disease. Neurotherapeutics 2004;1: 382-393.

$\square_{8}$ Björklund A, Dunnett SB: Dopamine neuron systems in the brain: an update. Trends Neurosci 2007;30:194-202.

-9 Sasaki T, Kotera J, Omori K: Transcriptional activation of phosphodiesterase 7B1 by dopamine D1 receptor stimulation through the cyclic AMP/cyclic AMP-dependent protein kinase/cyclic AMP-response element binding protein pathway in primary striatal neurons. J Neurochem 2004;89:474-483.

10 Franke H, Schelhorn N, Illes P: Dopaminergic neurons develop axonal projections to their target areas in organotypic co-cultures of the ventral mesencephalon and the striatum/prefrontal cortex. Neurochem Int 2003; 42:431-439.

11 Franke H, Illes P: Involvement of P2 receptors in the growth and survival of neurons in the CNS. Pharmacol Ther 2006; 109:297324.

$\$ 12$ Heine C, Wegner A, Grosche J, Allgaier C, Illes $\mathrm{P}$, Franke H: P2 receptor expression in the dopaminergic system of the rat brain during development. Neuroscience 2007; 149:165-181.

13 Song HJ, Poo MM: Signal transduction underlying growth cone guidance by diffusible factors. Curr Opin Neurobiol 1999;9:355363.

14 Tojima T, Kobayashi S, Ito E: Dual role of cyclic AMP-dependent protein kinase in neuritogenesis and synaptogenesis during neuronal differentiation. J Neurosci Res 2003;74: 829-837.

15 Cai D, Shen Y, De Bellard M, Tang S, Filbin MT: Prior exposure to neurotrophins blocks inhibition of axonal regeneration by MAG and myelin via a cAMP-dependent mechanism. Neuron 1999;22:89-101.

16 Francis SH, Turko IV, Corbin JD: Cyclic nucleotide phosphodiesterases: relating structure and function. Prog Nucleic Acid Res Mol Biol 2001;65:1-52.

17 Bender AT, Beavo JA: Cyclic nucleotide phosphodiesterases: molecular regulation to clinical use. Pharmacol Rev 2006;58:488520.

18 Conti M, Beavo J: Biochemistry and physiology of cyclic nucleotide phosphodiesterases: essential components in cyclic nucleotide signaling. Annu Rev Biochem 2007;76:481511.
19 Rutten K, Prickaerts J, Hendrix M, van der Staay FJ, Sik A, Blokland A: Time-dependent involvement of cAMP and cGMP in consolidation of object memory: studies using selective phosphodiesterase type 2, 4 and 5 inhibitors. Eur J Pharmacol 2007;558:107-112.

20 Stephenson DT, Coskran TM, Wilhelms $\mathrm{MB}$, Adamowicz WO, O’Donnell MM, Muravnick $\mathrm{KB}$, et al: Immunohistochemical localization of phosphodiesterase $2 \mathrm{~A}$ in multiple mammalian species. J Histochem Cytochem 2009;57:933-949.

21 Siuciak JA, Chapin DS, Harms JF, Lebel LA, McCarthy SA, Chambers L, et al: Inhibition of the striatum-enriched phosphodiesterase PDE10A: a novel approach to the treatment of psychosis. Neuropharmacology 2006;51: 386-396.

22 Chappie T, Humphrey J, Menniti F, Schmidt C: PDE10A inhibitors: an assessment of the current CNS drug discovery landscape. Curr Opin Drug Discov Devel 2009;12:458-467.

-23 Grauer SM, Pulito VL, Navarra RL, Kelly MP, Kelley C, Graf R, et al: Phosphodiesterase $10 \mathrm{~A}$ inhibitor activity in preclinical models of the positive, cognitive, and negative symptoms of schizophrenia. J Pharmacol Exp Ther 2009;331:574-590.

24 Nishi A, Snyder GL: Advanced research on dopamine signaling to develop drugs for the treatment of mental disorders: biochemical and behavioral profiles of phosphodiesterase inhibition in dopaminergic neurotransmission. J Pharmacol Sci 2010;114:6-16.

25 Höfgen N, Stange H, Schindler R, Lankau HJ, Grunwald C, Langen B, et al: Discovery of imidazo[1,5-a]pyrido[3,2-e]pyrazines as a new class of phosphodiesterase 10A inhibitors. J Med Chem 2010;53:4399-4411.

26 Paglia MJ, Mou H, Cote RH: Regulation of photoreceptor phosphodiesterase (PDE6) by phosphorylation of its inhibitory gamma subunit re-evaluated. J Biol Chem 2002;277: 5017-5023.

27 Vivas FA, Pestana RC, Ursin B: A new stabilized least-squares imaging condition. J Geophys Eng 2009;6:264-268.

28 Rudin LI, Osher S, Fatemi E: Nonlinear total variation based noise removal algorithms. Physica D: Nonlinear Phenomena 1992;60: 259-268.

29 Gonzalez RC, Woods RE: Digital Image Processing, ed 2. Prentice Hall International, 2001.

30 Otsu N: A threshold selection method from gray-level histograms. IEEE Trans Syst Man Cybern 1979;9:62-66.

31 Gomez-Urquijo SM, Hökfelt T, Ubink R, Lubec G, Herrera-Marschitz M: Neurocircuitries of the basal ganglia studied in organotypic cultures: focus on tyrosine hydroxylase, nitric oxide synthase and neuropeptide immunocytochemistry. Neuroscience 1999; 94:1133-1151.
32 Polleux F, Morrow T, Ghosh A: Semaphorin $3 \mathrm{~A}$ is a chemoattractant for cortical apical dendrites. Nature 2000;404:567-573.

33 Jaumotte JD, Zigmond MJ: Dopaminergic innervation of forebrain by ventral mesencephalon in organotypic slice co-cultures: effects of GDNF. Brain Res Mol Brain Res 2005;134:139-146.

34 Tamariz E, Díaz-Martínez NE, Díaz NF, García-Peña CM, Velasco I, Varela-Echavarría A: Axon responses of embryonic stem cell-derived dopaminergic neurons to semaphorins 3A and 3C. J Neurosci Res 2010;88: 971-980.

35 Voorn P, Kalsbeek A, Jorritsma-Byham B, Groenewegen HJ: The pre- and postnatal development of the dopaminergic cell groups in the ventral mesencephalon and the dopaminergic innervation of the striatum of the rat. Neuroscience 1988;25:857-887.

36 Van Staveren WCG, Steinbusch HWM, Markerink-Van Ittersum M, Repaske DR, Goy MF, Kotera J, et al: mRNA expression patterns of the cGMP-hydrolyzing phosphodiesterases types 2, 5, and 9 during development of the rat brain. J Comp Neurol 2003; 467:566-580.

37 Smoake JA, Song SY, Cheung WY: Cyclic 3',5'-nucleotide phosphodiesterase. Distribution and developmental changes of the enzyme and its protein activator in mammalian tissues and cells. Biochim Biophys Acta 1974;341:402-411.

38 Davis CW, Kuo JF: Ontogenetic changes in levels of phosphodiesterase for adenosine $3^{\prime}: 5^{\prime}$-monophosphate and glucosine $3^{\prime}: 5^{\prime}$ monophosphate in the lung, brain and heart from guinea pigs. Biochim Biophys Acta 1976;444:554-562.

39 Juilfs DM, Soderling S, Burns F, Beavo JA: Cyclic GMP as substrate and regulator of cyclic nucleotide phosphodiesterases (PDEs). Rev Physiol Biochem Pharmacol 1999;135: 67-104.

40 Mehats C, Andersen CB, Filopanti M, Jin SLC, Conti M: Cyclic nucleotide phosphodiesterases and their role in endocrine cell signaling. Trends Endocrinol Metab 2002;13: 29-35.

41 Fujishige K, Kotera J, Michibata H, Yuasa K, Takebayashi S, Okumura K, et al: Cloning and characterization of a novel human phosphodiesterase that hydrolyzes both cAMP and cGMP (PDE10A). J Biol Chem 1999;274: 18438-18445.

-42 Seeger TF, Bartlett B, Coskran TM, Culp JS, James LC, Krull DL, et al: Immunohistochemical localization of PDE10A in the rat brain. Brain Res 2003;985:113-126.

43 Xie Z, Adamowicz WO, Eldred WD, Jakowski AB, Kleiman RJ, Morton DG, et al: Cellular and subcellular localization of PDE10A, a striatum-enriched phosphodiesterase. Neuroscience 2006;139:597-607. 
44 Boess FG, Hendrix M, van der Staay F-J, Erb C, Schreiber R, van Staveren W, et al: Inhibition of phosphodiesterase 2 increases neuronal cGMP, synaptic plasticity and memory performance. Neuropharmacology 2004;47: 1081-1092.

-45 Deng C, Wang D, Bugaj-Gaweda B, De Vivo M: Assays for cyclic nucleotide-specific phosphodiesterases (PDEs) in the central nervous system (PDE1, PDE2, PDE4, and PDE10). Curr Protoc Neurosci 2007; Chapter 7:Unit 7.21.

-46 Bos LB: Epac proteins: multi-purpose cAMP targets. Trends Biochem Sci 2006;12:680686.

-47 Song H, Ming G, He Z, Lehmann M, McKerracher L, Tessier-Lavigne M, et al: Conversion of neuronal growth cone responses from repulsion to attraction by cyclic nucleotides. Science 1998;281:1515-1518.

48 Schmidt CJ, Chapin DS, Cianfrogna J, Corman ML, Hajos M, Harms JF, et al: Preclinical characterization of selective phosphodiesterase 10A inhibitors: a new therapeutic approach to the treatment of schizophrenia. J Pharmacol Exp Ther 2008;325:681-690.

49 Siuciak JA, McCarthy SA, Chapin DS, Fujiwara RA, James LC, Williams RD, et al: Genetic deletion of the striatum-enriched phosphodiesterase PDE10A: evidence for altered striatal function. Neuropharmacology 2006; 51:374-385
50 Nishi A, Kuroiwa M, Miller DB, O'Callaghan JP, Bateup HS, Shuto T, et al: Distinct roles of PDE4 and PDE10A in the regulation of cAMP/PKA signaling in the striatum. J Neurosci 2008;28:10460-10471.

51 Sotty F, Montezinho LP, Steiniger-Brach B, Nielsen J: Phosphodiesterase 10A inhibition modulates the sensitivity of the mesolimbic dopaminergic system to $\mathrm{D}$-amphetamine: involvement of the D1-regulated feedback control of midbrain dopamine neurons. J Neurochem 2009;109:766-775.

52 Chao MV: Neurotrophins and their receptors: a convergence point for many signalling pathways. Nat Rev Neurosci 2003;4:299-309.

53 Vaudry D, Stork PJS, Lazarovici P, Eiden LE: Signaling pathways for PC12 cell differentiation: making the right connections. Science 2002;296:1648-1649.

$54 \mathrm{Ng}$ YP, Wu Z, Wise H, Tsim KWK, Wong YH, Ip NY: Differential and synergistic effect of nerve growth factor and cAMP on the regulation of early response genes during neuronal differentiation. Neurosignals 2009;17:111-120.
55 Laasberg T, Pihlak A, Neuman T, Paves H, Saarma M: Nerve growth factor increases the cyclic GMP level and activates the cyclic GMP phosphodiesterase in PC1 2 cells. FEBS Lett 1988;239:367-370.

56 Engele J, Bohn MC: The neurotrophic effects of fibroblast growth factors on dopaminergic neurons in vitro are mediated by mesencephalic glia. J Neurosci 1991;11:3070-3078.

57 Nikkhah G, Odin P, Smits A, Tingström A, Othberg A, Brundin P, et al: Platelet-derived growth factor promotes survival of rat and human mesencephalic dopaminergic neurons in culture. Exp Brain Res 1993;92:516523.

58 Østergaard K, Jones SA, Hyman C, Zimmer $\mathrm{J}$ : Effects of donor age and brain-derived neurotrophic factor on the survival of dopaminergic neurons and axonal growth in postnatal rat nigrostriatal cocultures. Exp Neurol 1996;142:340-350.

59 Schatz DS, Kaufmann WA, Saria A, Humpel C: Dopamine neurons in a simple GDNFtreated meso-striatal organotypic co-culture model. Exp Brain Res 1999;127:270-278.

-60 Thompson LH, Grealish S, Kirik D, Björklund A: Reconstruction of the nigrostriatal dopamine pathway in the adult mouse brain. Eur J Neurosci 2009;30:625-638. 